University of Wollongong

Research Online

Faculty of Engineering and Information

Faculty of Engineering and Information

Sciences - Papers: Part A

Sciences

$1-1-2008$

Tangential sphere bounds on the ensemble performance of $M L$ decoded Gallager codes via their exact ensemble distance spectrum

Sheng Tong

Xidian Unviersity, sheng@uow.edu.au

Follow this and additional works at: https://ro.uow.edu.au/eispapers

Part of the Engineering Commons, and the Science and Technology Studies Commons

Research Online is the open access institutional repository for the University of Wollongong. For further information contact the UOW Library: research-pubs@uow.edu.au 


\title{
Tangential sphere bounds on the ensemble performance of ML decoded Gallager codes via their exact ensemble distance spectrum
}

\author{
Abstract \\ An efficient numerical approach to the exact ensemble distance spectrum of Gallager codes has been \\ developed by evaluating powers of polynomials. With the exact ensemble distance spectrum of Gallager \\ codes, tangential sphere upper bounds on their maximum likelihood $(\mathrm{ML})$ decoding performance over \\ binary input AWGN channels are investigated. Numerical results indicate improved bounds have been \\ obtained, better than Sason and Shamai's results (which are based on Gallager's upper bound on the \\ ensemble distance spectrum), especially in the error floor region. Furthermore, some critical properties of \\ Gallager codes, including typical minimum distance and the performance tradeoff in the waterfall and \\ error floor regions, have been considered.

\section{Keywords} \\ exact, their, via, codes, gallager, spectrum, decoded, distance, $\mathrm{ml}$, performance, ensemble, bounds, sphere, \\ tangential \\ Disciplines \\ Engineering | Science and Technology Studies

\section{Publication Details} \\ S. Tong, "Tangential sphere bounds on the ensemble performance of ML decoded Gallager codes via their \\ exact ensemble distance spectrum," in IEEE International Conference on Communications: Proceedings, \\ 2008, pp. 1150-1154.
}




\title{
Tangential Sphere Bounds on the Ensemble Performance of ML Decoded Gallager Codes via Their Exact Ensemble Distance Spectrum
}

\author{
Sheng Tong \\ Email: ts_xd@163.com
}

\begin{abstract}
An efficient numerical approach to the exact ensemble distance spectrum of Gallager codes has been developed by evaluating powers of polynomials. With the exact ensemble distance spectrum of Gallager codes, tangential sphere upper bounds on their maximum likelihood (ML) decoding performance over binary input AWGN channels are investigated. Numerical results indicate improved bounds have been obtained, better than Sason and Shamai's results (which are based on Gallager's upper bound on the ensemble distance spectrum), especially in the error floor region. Furthermore, some critical properties of Gallager codes, including typical minimum distance and the performance tradeoff in the waterfall and error floor regions, have been considered.
\end{abstract}

\section{INTRODUCTION}

Low-density parity-check (LDPC) codes were firstly introduced by Gallager in the early of 1960's [1,2], and rediscovered by MacKay and Neal [3] in the late of 1990's. In his seminal work, Gallager investigated a special class of regular LDPC codes, referred to as Gallager codes, and provided an analytical upper bound on their ensemble distance spectrum [2], which are further elaborated by Sason and Shamai [7]. Based on Gallager's ensemble distance spectrum upper bound, Sason and Shamai use tangential sphere bound [4] to investigate the ensemble performance of Gallager codes over binary input AWGN channels under maximum likelihood (ML) decoding [5], which further verifies their capacityapproaching performance at long block lengths.

In this paper, we improve Sason and Shamai's bounds by using the exact ensemble distance spectrum of Gallager codes. For this purpose, we develop an efficient approach to the exact ensemble distance spectrum of Gallager codes. With the exact ensemble distance spectrum, we use tangential sphere bounding technique to evaluate the ensemble performance of ML decoded Gallager codes over binary input AWGN channels. Tangential sphere bounds are upper bounds and depend on the distance spectrum of the considered code. Although there are several tighter but more involved bounds $[11,12]$, we still employ TSB for its simplicity.

The rest of the paper is organized as follows: in Section II, we briefly review the definition of Gallager codes and

The author is with State Key Lab. of ISN, Xidian Unviersity and Department of Electronic Engineering, City University of Hong Kong. This work was supported in part by the China High-Tech 863 Program under Grant 2006AA01Z267, and the National Natural Science Foundation of China under Grants 60496316, 60572059, and U0635003. then develop an efficient approach to their exact ensemble distance spectrum. In Section III, improved tangential sphere bounds based on the exact ensemble distance spectrum on the ensemble performance of ML decoded Gallager codes over binary input AWGN channels are presented. Furthermore, some properties of Gallager codes, including typical minimum distance and the performance tradeoff in the waterfall and error floor regions, are considered. Section IV concludes the paper.

\section{Calculation of Ensemble Distance Spectrum of Gallager Codes}

Following Gallager, an $(n, j, k)$ Gallager code is a length- $n$ block code, specified by a sparse parity-check matrix $\mathbf{H}$. It is shown in eqn.(1) that the parity-check matrix $\mathbf{H}$ consists of $j$ sub-matrices $\mathbf{H}^{i}(i=1,2, \cdots, j)$ of the same size, each containing a single 1 in each column and $k$ 1's in each row. The first sub-matrix $\mathbf{H}^{1}$ is constructed in a staircase form, i.e., the $k$ 1's in its $i$ th row are located from the $((i-1) k+1)$ th column to the $(i k)$ th column, as shown in eqn.(2).The other sub-matrices are just random permutations of the first one. Every sub-matrix $\mathbf{H}^{i}$ defines a super code $\mathcal{C}^{i}$, which can be viewed as the direct sum of $(n / k)$ same single parity-check (SPC) codes, each denoted as $(k, k-1,2)$. Thus, Gallager code can be interpreted as the intersection of the $j$ super codes.

$$
\mathbf{H}=\left(\begin{array}{c}
\mathbf{H}^{1} \\
\mathbf{H}^{2} \\
\vdots \\
\mathbf{H}^{j}
\end{array}\right)
$$

where

$$
\mathbf{H}^{1}=\left(\begin{array}{ccc}
\underbrace{1 \cdots 1}_{k} & & \\
& \ddots & \\
& & \underbrace{1 \cdots 1}_{k}
\end{array}\right)
$$

Now we consider the ensemble distance spectrum of $(n, j, k)$ Gallager codes. From above, the ensemble distance spectrum of Gallager codes can be obtained via a two-step procedure. We shall first outline the two-step procedure in the following and then discuss the two steps in details. 


\section{Calculation of the Ensemble Distance Spectrum of $(n, j, k)$ Gallager Codes}

1) Calculate the distance spectrum of each super code. Note that the other $(j-1)$ super codes are just random permutations of the first one. We only need to find the distance spectrum for the first super code $\mathcal{C}^{1}$.

2) Under the uniform interleaver assumption, the distance spectrum of Gallager codes can be easily derived from those of the super codes.

Firstly, the step 1) is considered. For a code $\mathcal{C}$ of length- $n$, denote its weight enumerating function (WEF) as

$$
A^{\mathcal{C}}(X)=\sum_{d=0}^{n} A_{d} X^{d}
$$

where $A_{d}$ is the number of codewords of weight- $d$. From eqn.(2) and the fact that the super code $\mathcal{C}^{1}$ is the direct sum of $n / k(k, k-1,2)$ SPC codes, the WEF of $\mathcal{C}^{1}$ can be obtained from that of a $(k, k-1,2)$ SPC code as follows.

$$
A^{\mathcal{C}^{1}}(X)=\left[A^{S P C}(X)\right]^{(n / k)},
$$

where $A^{S P C}(X)$ denotes the WEF of a $(k, k-1,2)$ SPC code, given by

$$
A^{S P C}(X)=\sum_{i=0}^{\lfloor k / 2\rfloor}\left(\begin{array}{c}
k \\
2 i
\end{array}\right) X^{2 i},
$$

where $\lfloor x\rfloor$ denotes the largest integer not greater than $x$.

From eqn.(4), it is known that the calculation of the distance spectrum of $\mathcal{C}^{1}$ only involves a power of a polynomial $A^{S P C}(X)$, which contains a relatively small number of terms for $k$ not greater than a few dozens. It is known that polynomial multiplication can be interpreted as vector convolution, which can be efficiently done using $\mathrm{FFT}^{1}$. However, due to the numerical problems in the calculation, this approach does not work well even for a block length of a few hundreds of bits.

Fortunately, there have been developed several efficient methods for evaluating powers in [9]. For simplicity, we use the "right-to-left binary method" to evaluate eqn.(4). For example, to evaluate $f(x)^{23}$, we first write the index 23 in its binary representation, i.e., 10111. Then replace each "1" by the pair of letters "SX", replace each "0" by "S", and delete the "SX" at the most left side. The obtained sequence is "SSXSXSX", which gives the rule for computing $f(x)^{23}$ by interpreting each " $\mathrm{S}$ " as squaring and each " $\mathrm{X}$ " as multiplying by $f(x)$. More clearly, we should successively compute $f(x)^{2}, f(x)^{4}, f(x)^{5}, f(x)^{10}, f(x)^{11}, f(x)^{22}, f(x)^{23}$. Generally, to evaluate $f(x)^{m}$ for a positive integer $m$, the required number of polynomial multiplications by the rightto-left binary method is $\left\lfloor\log _{2}(m)\right\rfloor+v(m)-1$, where $v(m)$ denotes the number of " 1 "'s in the binary representation of $m$. Thus, to evaluate eqn.(4), we should carry out $\left\lfloor\log _{2}(n / k)\right\rfloor+$ $v(n / k)-1$ times of polynomial multiplications.

\footnotetext{
${ }^{1}$ Note that the vector convolution used here is linear convolution. To avoid circular convolution, we must pad zero terms to $A^{S P C}(X)$ to the term of $X^{n}$ before applying an FFT
}

Once we get the WEF for the first super codes, we also get WEF's for the other super codes since they are just random permutations of the first super code. Thus, all super codes share the same WEF.

Now, we consider the step2). Under the uniform interleaver assumption, the probability of a weight- $d$ codeword of $\mathcal{C}^{1}$ being a codeword of another super code is $A_{d}^{\mathcal{C}^{1}} /\left(\begin{array}{l}n \\ d\end{array}\right)$. Thus, the average number of weight $d$ codewords in an $(n, j, k)$ Gallager code $\mathcal{C}$ is

$$
A_{d}^{\mathcal{C}}=\frac{\left[A_{d}^{\mathcal{C}^{1}}\right]^{j}}{\left(\begin{array}{l}
n \\
d
\end{array}\right)^{j-1}},
$$

Obviously, eqn.(6) gives the $d$-th term of the ensemble distance spectrum $A^{\mathcal{C}}$ for $(n, j, k)$ Gallager codes. Thus, we obtain the ensemble distance spectrum for Gallager codes.

\section{NUMERICAL RESULTS}

In his seminal work [2], Gallager also derived an upper bound on the ensemble distance spectrum of Gallager codes. The comparison between the exact ensemble distance spectrum and Gallager's upper bound on ensemble distance spectrum of Gallager codes is presented in Fig.1, for some $(n, 3,6)$ Gallager code ensembles with $n=108,504,1008,10008$. As already indicated in [4], the shaping of the distance spectrum of these ensembles of Gallager codes is rather typical for other block lengths and rates. Fig.1 shows that with the increase of code lengths Gallager's upper bounds get closer to the exact ensemble distance spectrum. It is seen from Fig.1 that Gallager's upper bound just lays above the exact ensemble distance spectrum of $(10008,3,6)$ Gallager codes. Hence, for long block lengths, performance in the waterfall region estimated via Gallager's distance spectrum upper bound become very close to those obtained from the exact distance spectrum by using the tangential sphere bounding technique, as shown in Fig.3(b). However, there is still a observable difference at the low weight part, which determines a significant performance difference in the error floor region, which is also indicated in Fig.3(b).

Gallager also proved that for every pair of $(j, k)$ with $j \geq 3$, there is a normalized Hamming threshold weight such that for Hamming weight below it, the exponent of the upper bound on the ensemble distance spectrum is negative [2]. Thus, for large block lengths, the typical minimum distance increases linearly with block length for $(n, j, k)$ Gallager codes. In this paper, with the exact ensemble distance spectrum $A^{\mathcal{C}}$ of $(n, j, k)$ Gallager codes, we define their typical minimum distance as the minimum positive integer $d$ such that $A_{d}^{\mathcal{C}} \geq 1$, i.e.,

$$
d_{\min }^{*}=\min \left\{d \mid d>0, A_{d}^{\mathcal{C}} \geq 1\right\}
$$

Typical minimum distance of Gallager codes for different pairs of $(j, k)$ and block lengths are shown in Fig.2. From Fig.2, it is seen that for a fixed pair of $(j, k)$, the typical minimum distance grows almost linearly with the block length. Moreover, for a fixed ratio of $j / k$ (i.e., keeping the code rate constant) and a fixed block length $n$, increasing the values of $j$ and $k$ will effectively increase the typical minimum distance. 

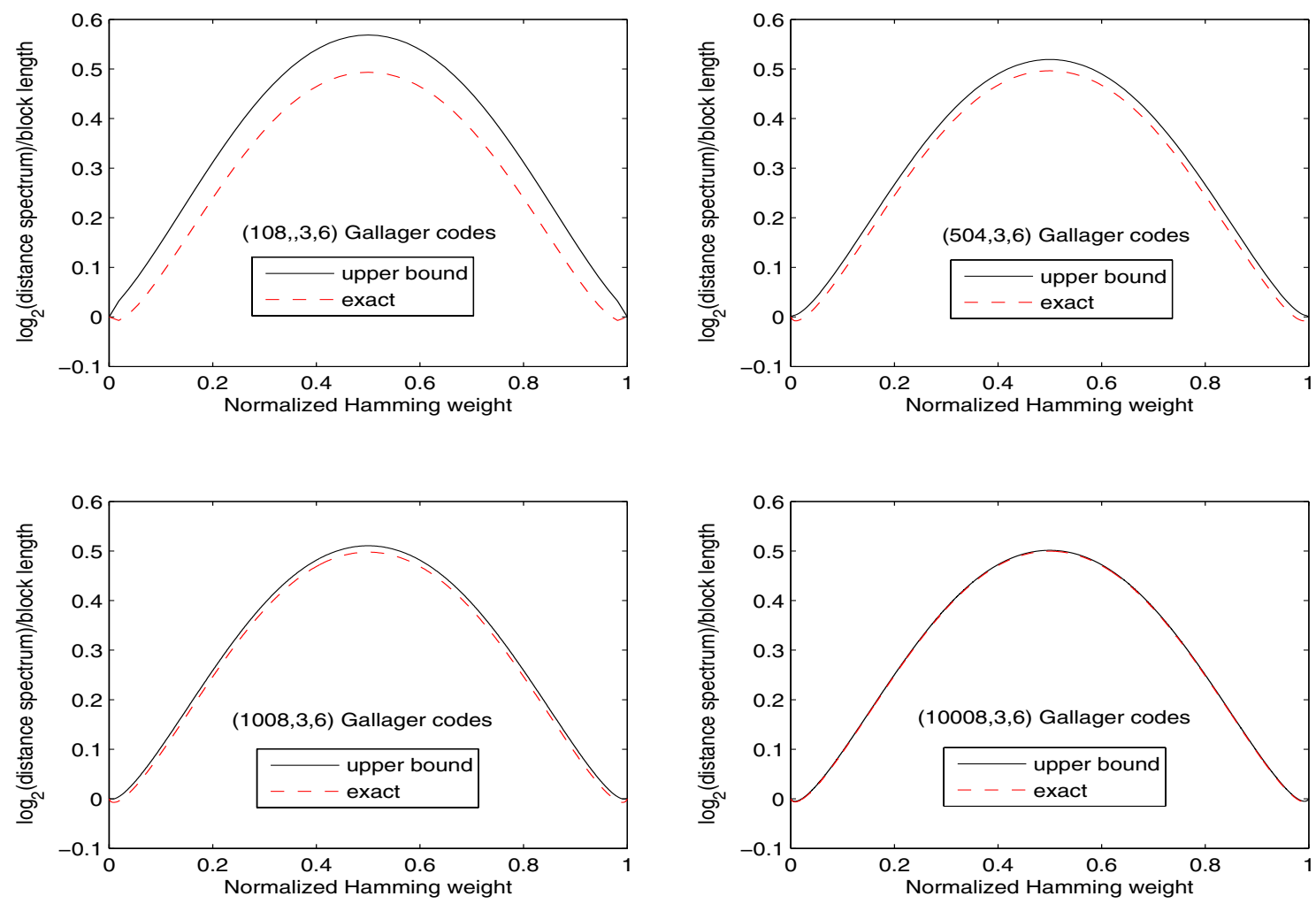

Fig. 1. Comparison of exact ensemble distance spectrum and Gallager's upper bound on ensemble distance spectrum for Gallager codes.

TABLE I

NORMALIZED TYPICAL MINIMUM DISTANCE FOR GALLAGER CODES.

\begin{tabular}{c|r|r|r|r|r}
\hline$(j, k)$ & $(3,6)$ & $(4,8)$ & $(5,10)$ & $(6,12)$ & $(10,20)$ \\
\hline$\delta_{j, k}$ & 0.0235 & 0.0633 & 0.0847 & 0.0958 & 0.1082 \\
\hline
\end{tabular}

These numerical results agree well with Gallager's analytical analysis. The ratios $\delta_{j, k}$ of typical minimum distance to block length for given pairs of $(j, k)$ are listed in Table I. These $\delta_{j, k}$ 's are obtained by curve fitting using the least square method with the data provided in Fig.2, which can be interpreted as the normalized typical minimum distance with respect to the block length.

Tangential sphere upper bounds on the ML decoding performance of some ensembles of $(n, j, k)$ Gallager codes with rate- 0.5 are presented in Fig.3(a) and (b). Fig.3(a) shows that tangential sphere bounds via exact ensemble distance spectrum improve greatly over those based on Gallager's upper bound on ensemble distance spectrum in both the waterfall region and the error floor region for medium block lengths. By comparing Fig.3(a) and (b), we see that with the increase of block length, the improvement in the waterfall region decreases, while there is still a significant improvement in the error floor region. It is also seen from Fig.3(a) and (b) that for a fixed block length and ratio of $j / k$, the performance of Gallager codes improves

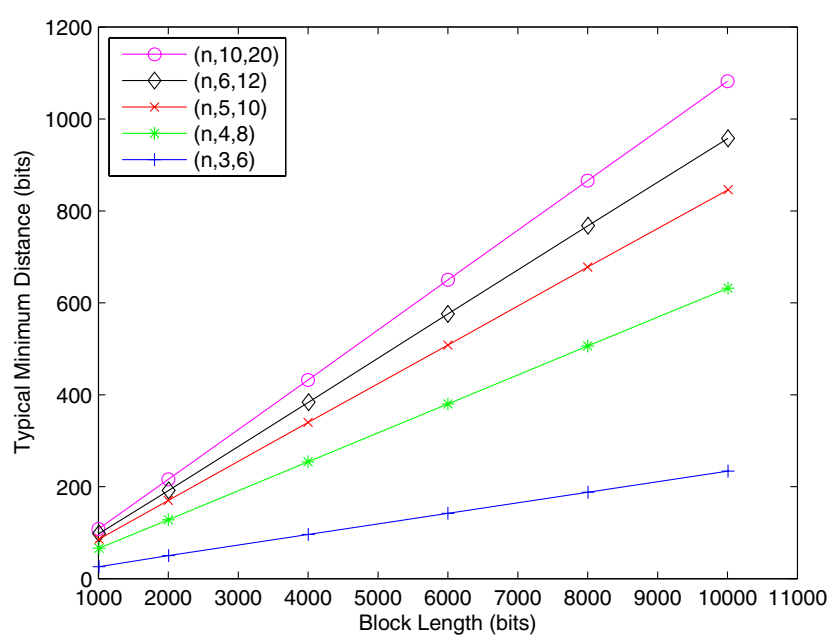

Fig. 2. Typical minimum distance of Gallager codes versus the block lengths.

with the increase of $j$ and $k$. Moreover, Fig.3(a) and (b) also demonstrate the effect of block length on the performance of Gallager codes for a fixed pair of $(j, k)$. For example, for the case of $j=6$ and $k=12$, the values of $E_{b} / N_{0}$ in decibel required by tangential sphere bounds on block error rate via 

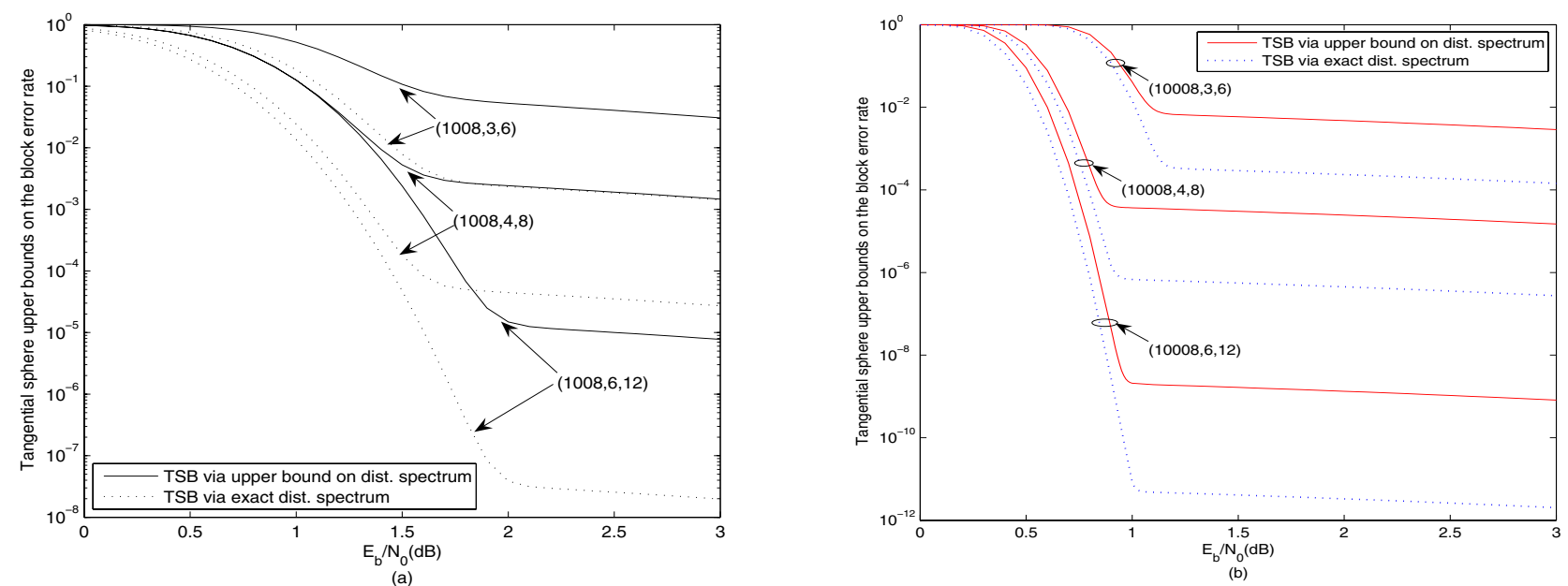

Fig. 3. Tangential sphere upper bound on the block error rate of Gallager codes used over a binary-input AWGN channel.(a) $n=1008$;(b) $n=10008$.

exact ensemble distance spectrum for achieving a block error rate of $10^{-5}$ are $1.60 \mathrm{~dB}$ and $0.74 \mathrm{~dB}$ for $n=1008$ and $n=$ 10008 , respectively.

It has been pointed out in [5] that for a fixed block length and a fixed ratio of $j / k$, there is only a slight improvement in the ensemble performance in the waterfall region by increasing the value of $j$ larger than 6 (see Fig.3(b)). Hence, following [5], we focus on $(n, j, k)$ Gallager codes with $j=6$ in the following. We consider Gallager codes of a variety of rates and block lengths with $j=6$ under ML decoding over binary input AWGN channels. The upper bounds on the values of $E_{b} / N_{0}$ in decibel (from tangential sphere bound analysis) for Gallager codes to achieve a block error rate of $10^{-5}$ are summarized in Table II, demonstrating their potential exceptional performance. Note that the true rate of $(n, j, k)$ Gallager codes is slightly larger than the design rate $(1-j / k)$, while for large block lengths the true rate is very close to the design rate. Hence, the Shannon limits used in Table II are for the design rates over binary input AWGN channels.

By increasing $j$ for a fixed ratio of $j / k$, we observe an interesting performance tradeoff between the waterfall region and error floor region, as demonstrated in Fig.4. From Fig.4(b), it is seen that by increasing $j$ from 6 to 12 for a fixed ratio of $j / k=0.5$, the error floor has been lowered by several orders of magnitude at the expense of a slight performance degradation in the waterfall region, which implies a performance tradeoff between the waterfall region and error floor region. This phenomenon can be explained by observing their respective ensemble distance spectrum in Fig.4(a). Fig.4(a) shows that $(1008,12,24)$ Gallager codes typically have a much larger minimum distance than $(1008,6,12)$, thus exhibiting a significant improvement in the error floor performance. However, the medium weight part in ensemble distance spectrum of $(1008,12,24)$ lies above that of $(1008,6,12)$ Gallager codes, which contributes a lot to the waterfall region performance. Thus, $(1008,6,12)$ Gallager codes beat slightly $(1008,12,24)$ Gallager in the waterfall region.

\section{CONCLUSions}

By developing an efficient approach to ensemble distance spectrum of Gallager codes, we have investigated the ensemble performance of ML decoded Gallager codes over binary input AWGN channels using the tangential sphere bounding technique. Our conclusions are summarized as follows:

1) With the increase of block lengths, the difference between Gallager's upper bound on ensemble distance spectrum and the exact distance spectrum of Gallager codes decreases. However, it is still observable in the low weight part, which determines that there remains a significant performance difference in the error floor region, as evidenced in Fig.3(b).

2) For Gallager code ensembles with a fixed pair of $(j, k)$, their typical minimum distance grows almost linearly with block lengths, which agrees well with Gallager's ananlytical results based on his upper bound on distance spectrum.

3) For a fixed ratio of $j / k$, by increasing the column weight $j$ to a relatively large value (say a few dozens), a performance tradeoff between the waterfall region and error floor region can be observed (see Fig.4(b)).

4) ML performance evaluation of Gallager codes using the tangential sphere bounds shows that increasing the column weight $j$ for a fixed ratio of $j / k$ and a constant block length will greatly improve the performance of Gallager codes, especially in the error floor region. However, for suboptimal and practical iterative decoders, the density evolution analysis [8] shows that for regular LDPC codes the column weight of $j=3$ works the best. A promising solution to this dilemma of binary Gallager codes is non-binary codes. More clearly, in the binary representation of the parity check matrix of an LDPC code over GF $(q)$, every non-binary element in the parity check matrix can be replaced by a small binary 
This full text paper was peer reviewed at the direction of IEEE Communications Society subject matter experts for publication in the ICC 2008 proceedings.

TABLE II

VALUE OF $E_{b} / N_{0}$ REQUIRED FOR TANGENTIAL SPHERE UPPER BOUND ON BLOCK ERROR RATE OF $10^{-5}$ WITH ML DECODING FOR $(n, j, k)$ GALLAGER CODE ENSEMBLE WITH $j=6$ (ASSUMING BPSK MODULATION AND AWGN CHANNELS). BOTH VALUE OF $E_{b} / N_{0}$ VIA EXACT ENSEMBLE DISTANCE SPECTRUM (LISTED BEFORE VIRGULES) AND THOSE VIA DISTANCE SPECTRUM UPPER BOUND (LISTED AFTER VIRGULES) ARE GIVEN.

\begin{tabular}{|c|c|c|c|c|c|}
\hline $\begin{array}{l}\text { The number of ones }(k) \\
\text { in each row of the parity } \\
\text { matrix } \mathbf{H} \text { of the code } \\
\text { ensemble and design code } \\
\text { rate (R) (Shannon limit) }\end{array}$ & $\begin{array}{r}k=8 \\
R=0.250 \\
(-0.79 \mathrm{~dB})\end{array}$ & $\begin{array}{r}k=10 \\
R=0.400 \\
(-0.24 \mathrm{~dB})\end{array}$ & $\begin{array}{r}k=12 \\
R=0.500 \\
(0.19 \mathrm{~dB})\end{array}$ & $\begin{array}{r}k=18 \\
R=0.667 \\
(1.06 \mathrm{~dB})\end{array}$ & $\begin{array}{r}k=24 \\
R=0.750 \\
(1.63 \mathrm{~dB})\end{array}$ \\
\hline The block length & \multicolumn{5}{|c|}{$\begin{array}{l}\text { Value of } E_{b} / N_{0}(\mathrm{~dB}) \text { via exact dist. } \\
\text { spectrum / those via dist. spectrum upper bound }\end{array}$} \\
\hline$n=5,040$ bits & $\begin{array}{r}0.07 \mathrm{~dB} / \\
0.20 \mathrm{~dB}\end{array}$ & $\begin{array}{r}0.50 \mathrm{~dB} / \\
0.59 \mathrm{~dB}\end{array}$ & $\begin{array}{r}0.91 \mathrm{~dB} / \\
1.00 \mathrm{~dB}\end{array}$ & $\begin{array}{r}1.85 \mathrm{~dB} / \\
1.93 \mathrm{~dB}\end{array}$ & $\begin{array}{r}2.48 \mathrm{~dB} / \\
2.57 \mathrm{~dB}\end{array}$ \\
\hline$n=10,080$ bits & $\begin{array}{l}-0.17 \mathrm{~dB} / \\
-0.10 \mathrm{~dB}\end{array}$ & $\begin{array}{r}0.31 \mathrm{~dB} / \\
0.36 \mathrm{~dB}\end{array}$ & $\begin{array}{r}0.74 \mathrm{~dB} / \\
0.79 \mathrm{~dB}\end{array}$ & $\begin{array}{l}1.70 \mathrm{~dB} / \\
1.74 \mathrm{~dB}\end{array}$ & $\begin{array}{l}2.35 \mathrm{~dB} \\
2.39 \mathrm{~dB}\end{array}$ \\
\hline$n=20,160$ bits & $\begin{array}{r}-0.35 \mathrm{~dB} / \\
-0.30 \mathrm{~dB} \\
\end{array}$ & $\begin{array}{r}0.17 \mathrm{~dB} / \\
0.20 \mathrm{~dB}\end{array}$ & $\begin{array}{r}0.62 \mathrm{~dB} / \\
0.65 \mathrm{~dB}\end{array}$ & $\begin{array}{r}1.60 \mathrm{~dB} / \\
1.62 \mathrm{~dB}\end{array}$ & $\begin{array}{r}2.25 \mathrm{~dB} / \\
2.27 \mathrm{~dB}\end{array}$ \\
\hline$n=40,320$ bits & $\begin{array}{r}-0.47 \mathrm{~dB} / \\
-0.45 \mathrm{~dB} \\
\end{array}$ & $\begin{array}{r}0.08 \mathrm{~dB} / \\
0.09 \mathrm{~dB} \\
\end{array}$ & $\begin{array}{r}0.53 \mathrm{~dB} / \\
0.55 \mathrm{~dB} \\
\end{array}$ & $\begin{array}{r}1.52 \mathrm{~dB} / \\
1.53 \mathrm{~dB} \\
\end{array}$ & $\begin{array}{r}2.17 \mathrm{~dB} / \\
2.19 \mathrm{~dB} \\
\end{array}$ \\
\hline
\end{tabular}

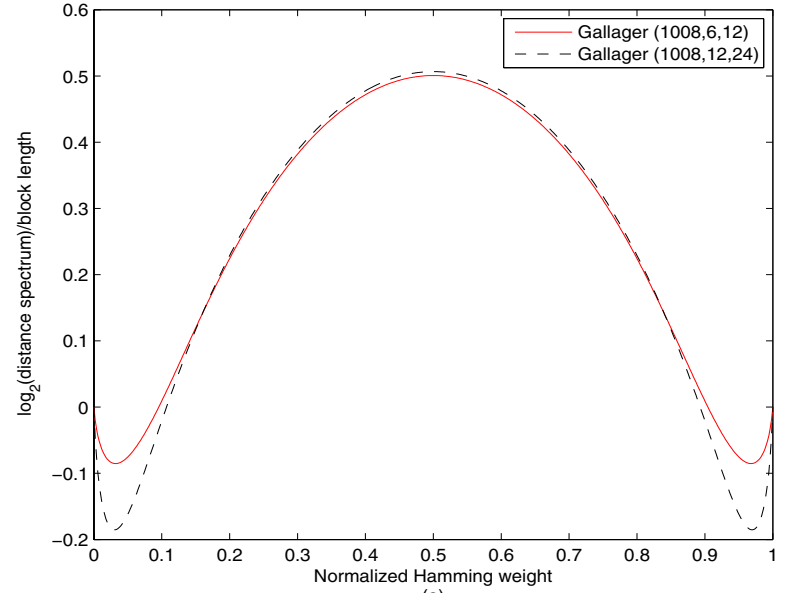

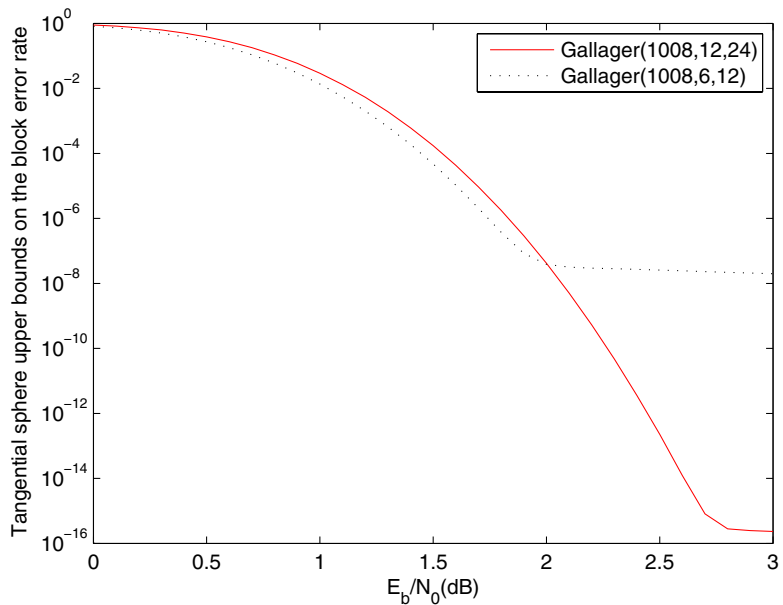

(b)

Fig. 4. Illustration of the performance tradeoff between the waterfall region and error floor region for two rate- 0.5 Gallager code ensembles.a) distance spectrum; b) tangential sphere upper bound on block error rate.

square matrix ${ }^{2}$, in which some columns may contain several ones, thus increasing the average column weight, while in the representation of the parity check matrix over $\operatorname{GF}(q)$ the column weight can be still very small and suitable for iterative decoders. This implies we can construct non-binary LDPC codes which perform well for both ML decoder and iterative decoders [10].

\section{REFERENCES}

[1] R. G. Gallager, "Low density parity check codes," IRE Trans. Inform. Theory, vol. IT-8, pp. 21-28, Jan. 1962.

[2] R. G. Gallager, Low Density Parity Check Codes. Cambridge, MA: M.I.T Press, 1963.

[3] D. J. C. Mackay and R. M. Neal, "Near Shannon limit performance of low density parity check codes," Electron. Lett., vol. 32, pp. 1645-1646, Aug. 1996.

[4] G. Poltyrev, "Bounds on the decoding error probability of binary linear codes via their spectra," IEEE Trans. Inform. Theory, vol. 40, pp. 12841292, July 1994.

\footnotetext{
${ }^{2}$ Note that all these binary square matrices and the all zero matrix of the same size form the finite field, $\operatorname{GF}(q)$.
}

[5] I. Sason and S. Shamai (Shitz), "Improved upper bounds on the ensemble performance of ML decoded low density parity check codes," IEEE Commun. Lett., vol.4, no.3, pp.89-91, Mar. 2000.

[6] I. Sason and S. Shamai (Shitz), "Improved upper bounds on the decoding error probability of parallel and serial concatenated turbo codes via their ensemble distance spectrum," IEEE Trans. Inform. Theory, vol. 46, pp.2447, Jan. 2000.

[7] I. Sason and S. Shamai (Shitz), "Tangential sphere bounds on the ensemble performance of ML decoded low density parity check codes," in Proc. of the 3rd ITC Conference on Source and Channel Coding, Jan., 2000.

[8] T. J. Richardson and R. L. Urbanke, "The capacity of low-density paritycheck. codes under message-passing decoding," IEEE Trans. Inform. Theory, vol. 47, no. 2, pp. 599-618, Feb. 2001.

[9] D. E. Knuth, The Art of Computer Programming. Vol.2: Seminumerical Algorithms, (3rd edition), pp.461, Addison-Wesley, 1998.

[10] X.-Y. Hu and E. Eleftheriou, "Binary representations of cycle tannergraph $G F\left(2^{b}\right)$ codes," in Proc. Intern. Conf. on Commun.,(ICC'04), vol.1, pp. 528-532, Paris, June, 2004.

[11] S. Yousefi and A.K. Khandani, "A new upper bound on the ML decoding error probability of linear binary block codes in AWGN interference," IEEE Trans. Inform. Theory, vol.50, no. 12, pp. 3026-3036, Dec. 2004.

[12] A. Mehrabian and S. Yousefi, "Improved tangential sphere bound on the ML decoding error probability of linear binary block codes in AWGN and block fading channels," IEE Proc. Commun., vol.153, no.6, pp.885-893, Dec. 2006. 